\title{
Patient Satisfaction of the Quality of Patient Teaching in Khatam Alanbia Hospital
}

\author{
Pirhosein Kolivand, Maryam Ali Hakami, Fateme Eghbali, Zahra Monjamed*, Farshad Sharifi
}

Shefa Neuroscience Research Center, Khatam Alanbia Hospital, Tehran, Iran

\section{A BSTRACT}

Introduction: Patient teaching is one of the important roles and independent responsibilities of nurses which needs special attention. Patient satisfaction is one of the important criteria to determine the quality of health care. Among different elements, patient teaching plays a crucial role in patient satisfaction. This study was aimed to determine the patient satisfaction of quality of patient teaching in Khatam Alanbia Hospital. Materials and Methods: This research is a descriptive- cross sectional study, which 595 patients from all wards (except Emergency and OPD) of Khatam Hospital were selected in 2015. The tool of data collection was questionnaire of patient satisfaction of quality of patient teaching and data analysis were done through descriptive and deferential statistics, such as Pearson Chi-square. Results: Most of the patients $(92.2 \%)$ had a high level of satisfaction towards quality of patient teaching. There was no correlation between patient satisfaction and sex, age as well as the level of education. However, a positive correlation between wards in which patients were hospitalized and patient satisfaction was observed. The highest level of satisfaction (100\%) was reported by patients hospitalized in obstetrics and gynecology department. Conclusion: The data revealed that patient satisfaction from quality of patient teaching may be a valuable indicator for determination of quality of services among different departments within a hospital.

\section{Key words:}

1. Patient Education as Topic

2. Patient Satisfaction

3. Nursing Care

*Corresponding Author: Zahra Monjamed

E-mail: monjamed2010@yahoo.com 


\title{
رضايتمندى بيمار از كيفيت آموزش بيمار در بيمارستان خاتمالانبياء
}

\author{
"يير حسين كوليوند، مريم على حكمى، فاطمه اقبالى، زهرا منجمد"، فرشاد شريفى \\ مركز تحقيقات علوم اعصاب شفا، بيمارستان خاتمالانبياء، تهران، ايران
}

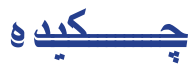

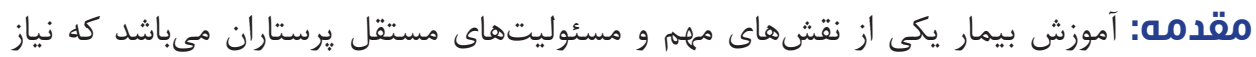

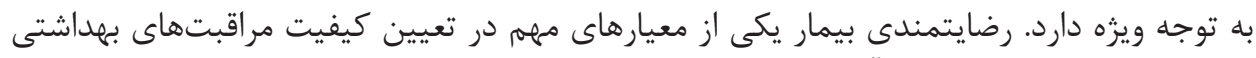

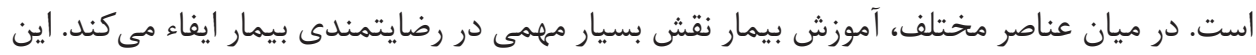

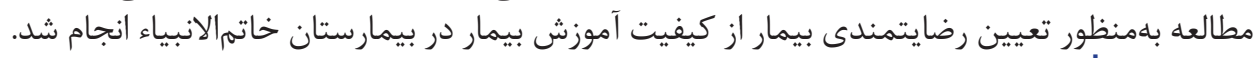

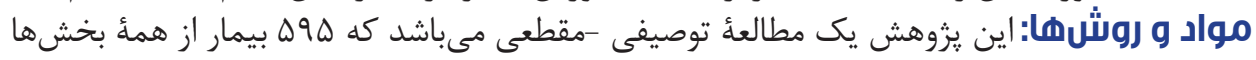

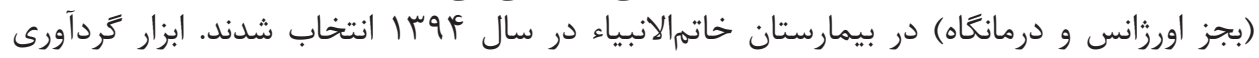

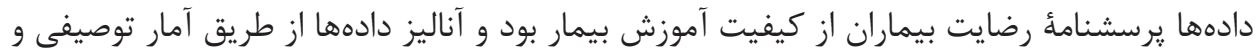

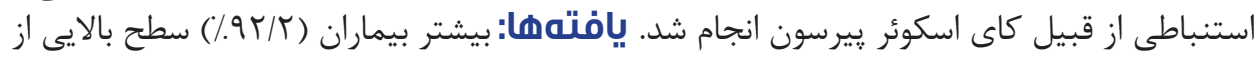

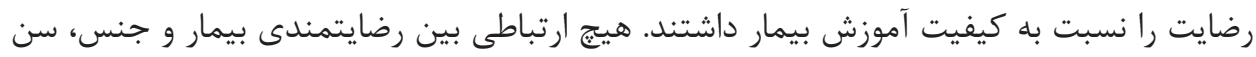

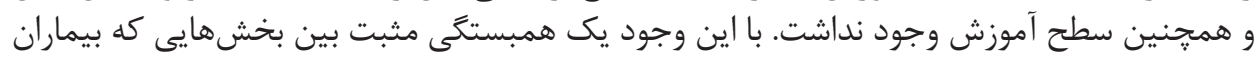

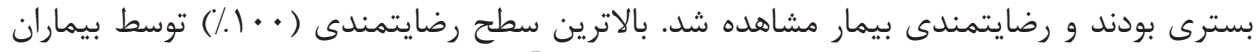

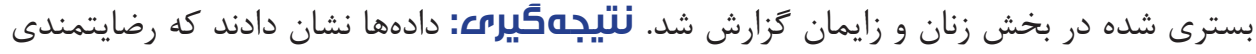

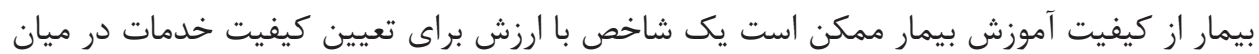
بخشهاى مختلف در يك بيمارستان باشد.

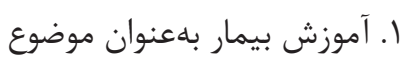

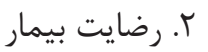

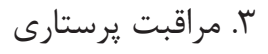




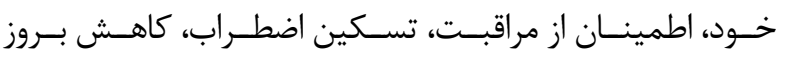

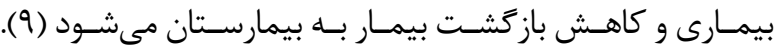

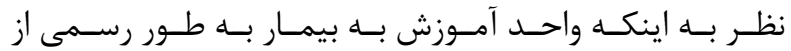

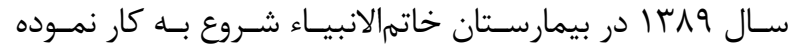

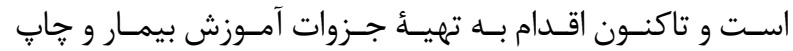

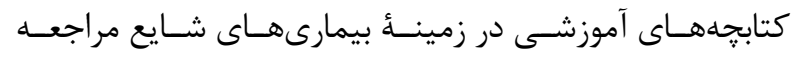

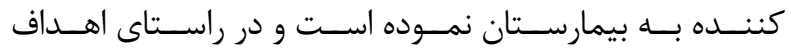

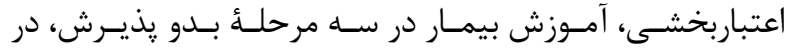

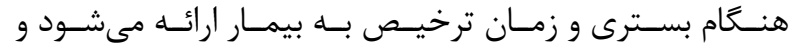

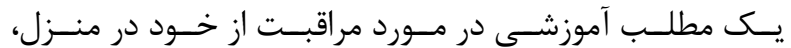

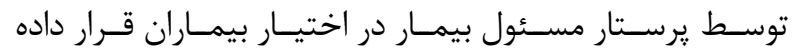

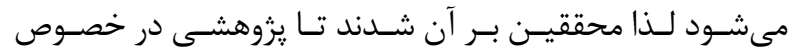

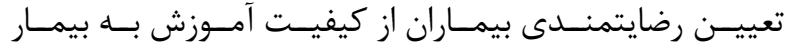

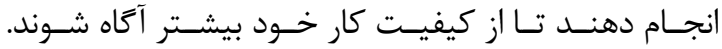

مواد و روشها

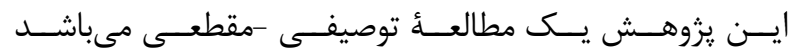

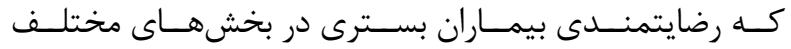

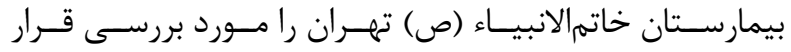

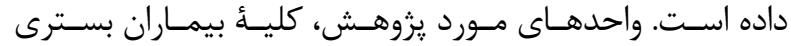

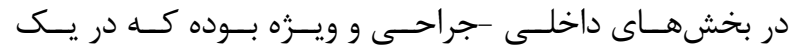

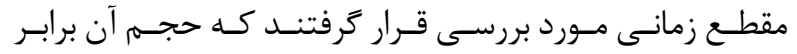

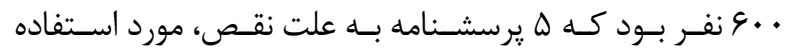

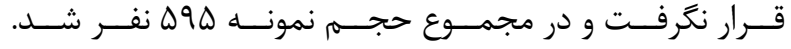

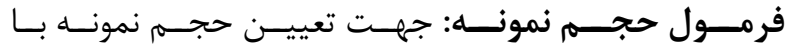

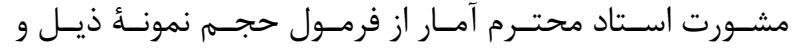

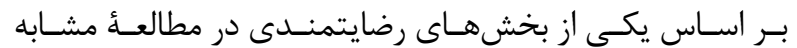

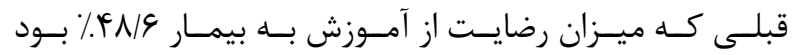

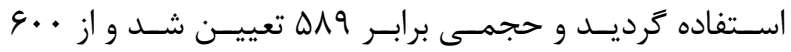

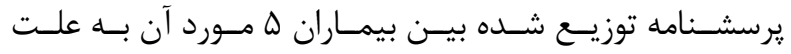

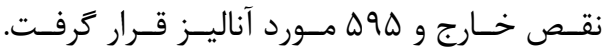

$$
\mathbf{n}=\frac{(\mathbf{P}(1-\mathbf{P}))}{\mathbf{m}^{r}} \times \frac{\mathbf{Z}_{\boldsymbol{\alpha}}}{r}
$$

روش نمونهَيرى

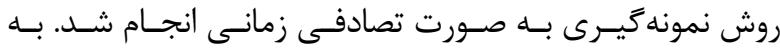

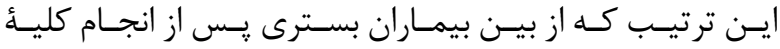

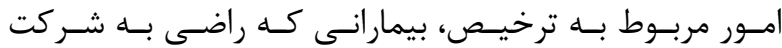

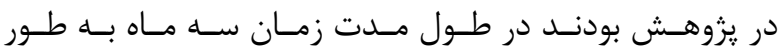

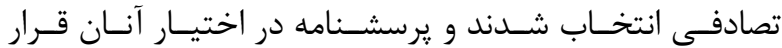

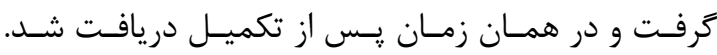

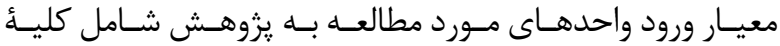

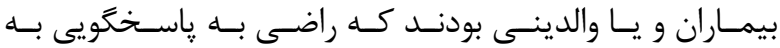

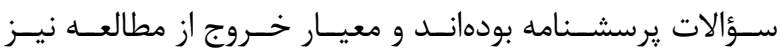

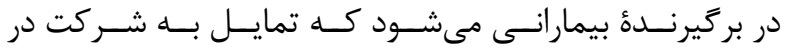
يزورهـش نداشـتند
مقدمه

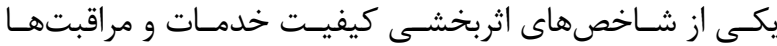

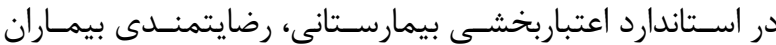

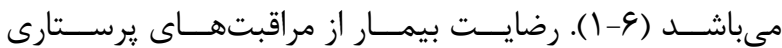

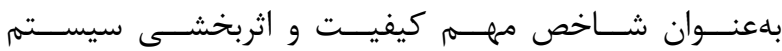

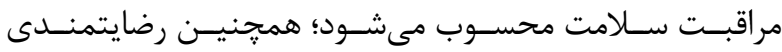

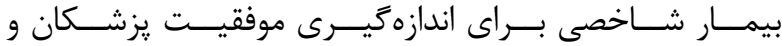

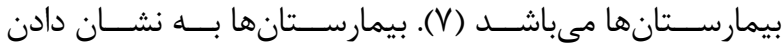

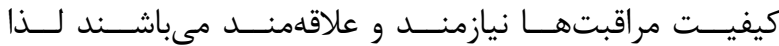

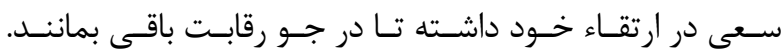

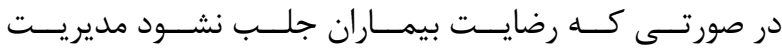

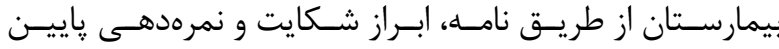

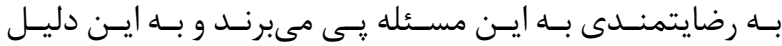

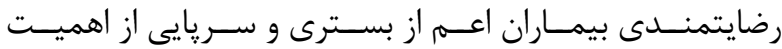

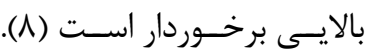

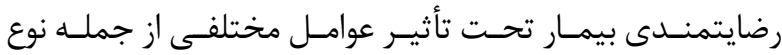

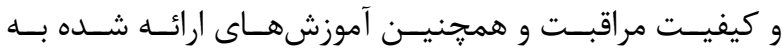

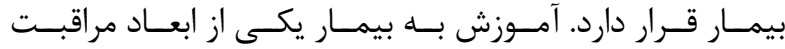

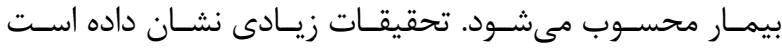

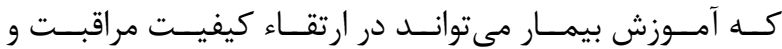

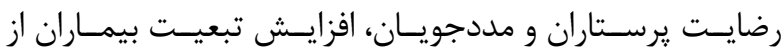

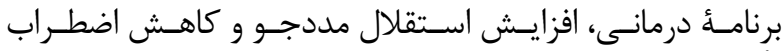

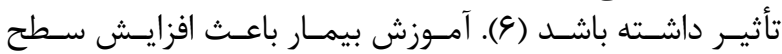

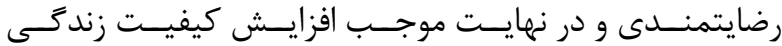

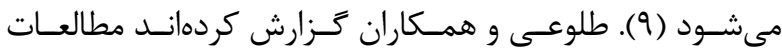

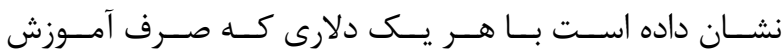

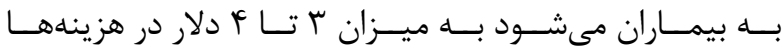

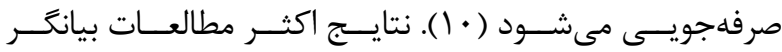

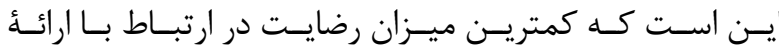

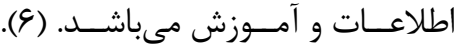

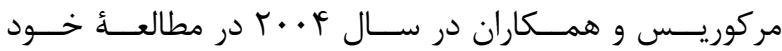

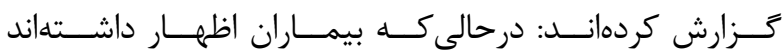

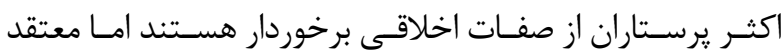

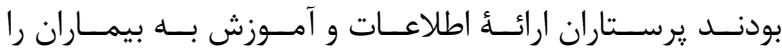

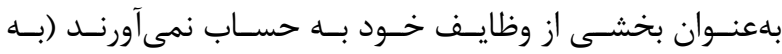

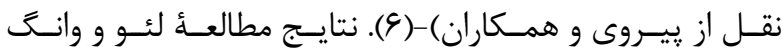

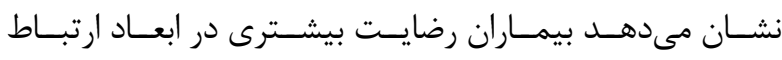

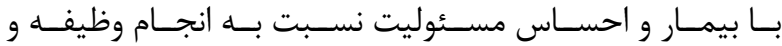

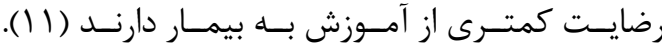

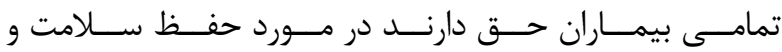

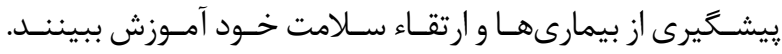

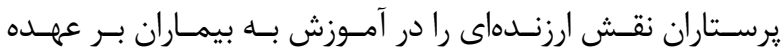

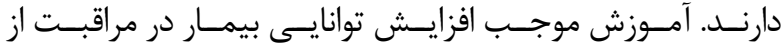

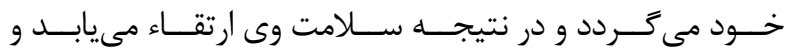

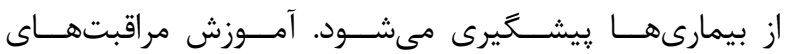

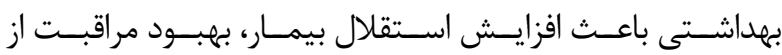




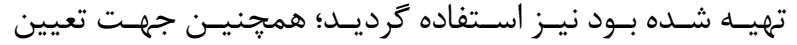

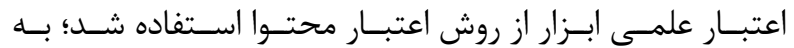

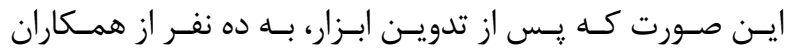

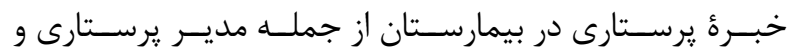

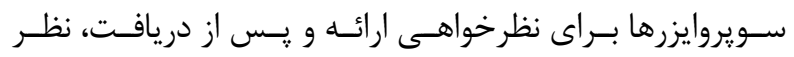

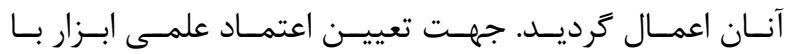

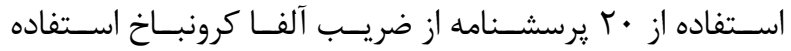

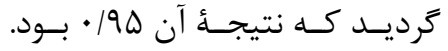
تجزيه و تحليل دادهها

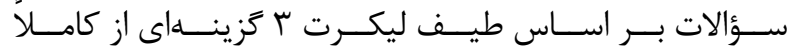

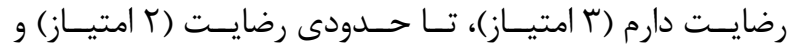

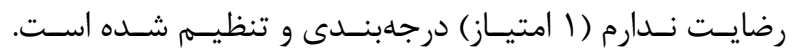

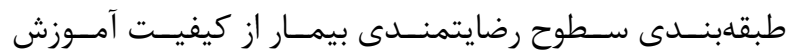

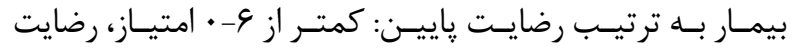

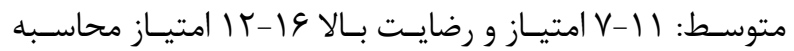

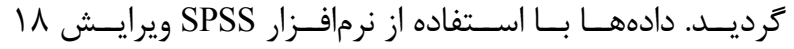

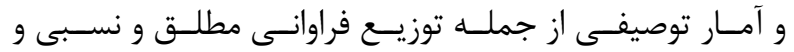

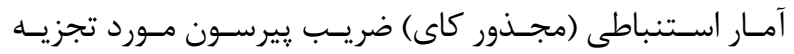

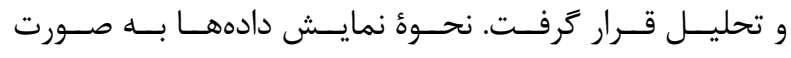

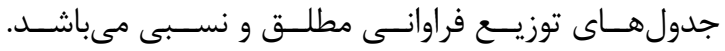

يافته ها

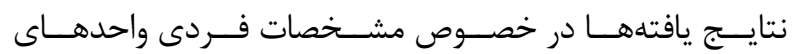

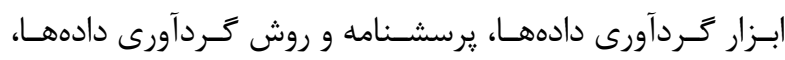

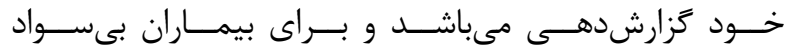

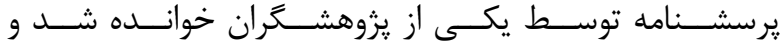

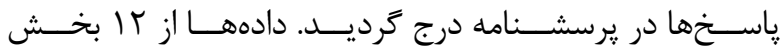

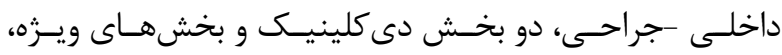

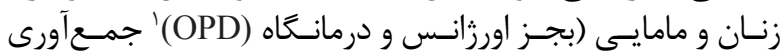

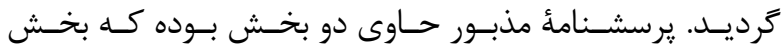

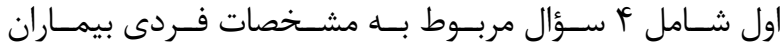

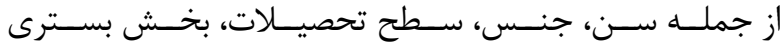

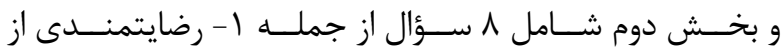

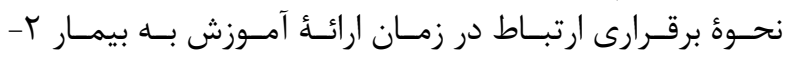

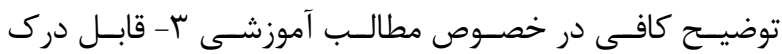

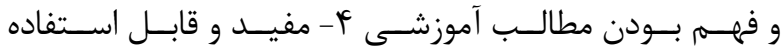

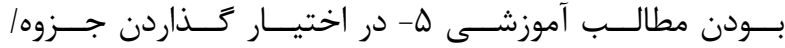

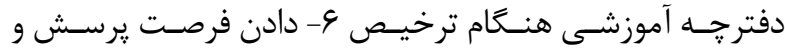

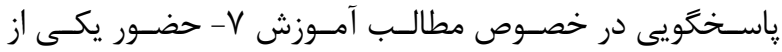

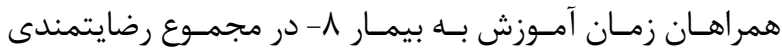

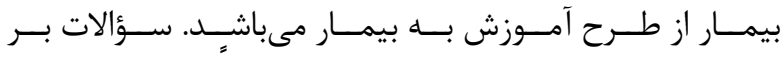

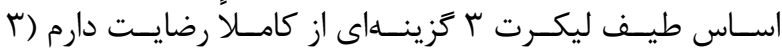

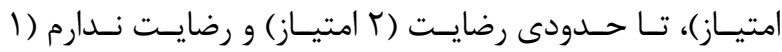

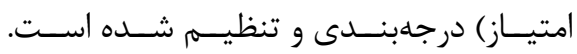

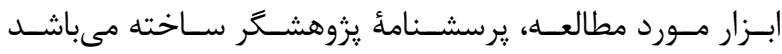

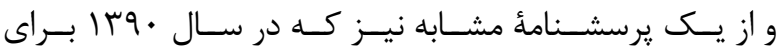

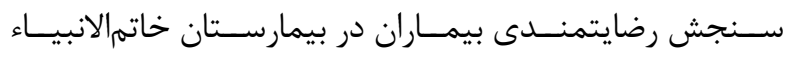

جدول ا- توزيع فراوانى مطلق و نسبى بيماران بر حسب مشخصات جمعيتشناختى و بخش بسترى.

\begin{tabular}{|c|c|c|c|}
\hline ل درصد & تعداث & متخير مورد مطالعه & \\
\hline$\Delta Q / T$ & rff & 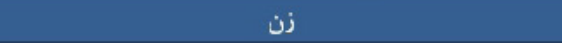 & \multirow{4}{*}{ 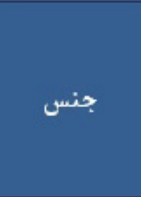 } \\
\hline$f \cdot / \Lambda$ & TrV & 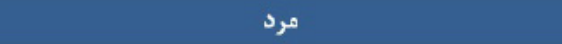 & \\
\hline- & 14 & بدون باسخ & \\
\hline $1 \cdots$ & $\Delta १ \Delta$ & جمع - la & \\
\hline $11 / \mathrm{V}$ & at & زير هץ سال & \multirow{6}{*}{ سن (سال) } \\
\hline ir & 99 & $r \cdot-r q$ & \\
\hline$r \cdot / \tau$ & $1 \cdot 1$ & $F+-F q$ & \\
\hline$\Delta F / q$ & ห१। & بيشتر از • +ه+ & \\
\hline- & 90 & بدون باسخ & \\
\hline $1 \ldots$ & $\Delta 90$ & جمع & \\
\hline $14 / 9$ & $y_{\Delta}$ & 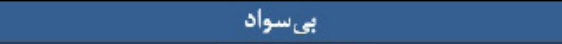 & \multirow{7}{*}{ تحصيلات } \\
\hline $14 / 9$ & $y_{\Delta}$ & راهنمايى اسيكل & \\
\hline $9 / \mathrm{V}$ & $\Delta \cdot$ & ديبرستانى & \\
\hline$r \cdot 10$ & 109 & ديبلم & \\
\hline$r \cdot / 9$ & lor & كاردانى، كارشناسى و بالاتر & \\
\hline- & AT & بدون باسخ & \\
\hline $1 \cdots$ & $\Delta १ \Delta$ & 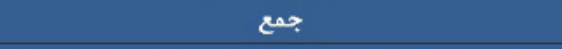 & \\
\hline$r \cdot / T$ & $1 \pi \cdot$ & بخش هاى جراحى مردانه (بخش 1- + - ه) و دى كلينـ & \multirow{7}{*}{ بخش بسترى } \\
\hline$r q / 1$ & Trt & بخشهاى جراحى زنانه (1r-11-1•-9-9B-rA-rB-1) & \\
\hline $1 \cdot 11$ & s. & بخش هانى داخلى (بخش V و (9A) & \\
\hline$r 1 / 9$ & 14. & بخشهاى ويره (CCU1-r-r-F-NICU-ICUOH) & \\
\hline$A / \%$ & $\Delta)$ & بخش زنان و زايمأن (مامايى) & \\
\hline- & r & بدون ياسخ & \\
\hline $1 \cdots$ & $\Delta १ \Delta$ & جمع & \\
\hline
\end{tabular}


بحث و نتيجه

آمــوزش بــه بيمــار داراى اثــرات بســيارى اســت از جملـــهـ

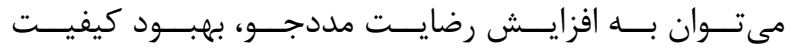

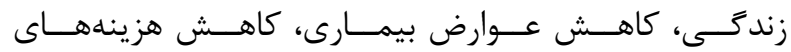

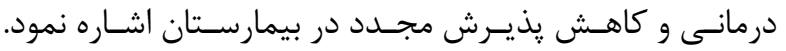

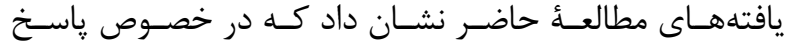

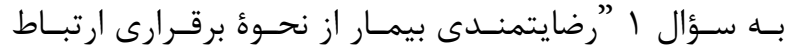

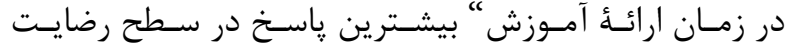

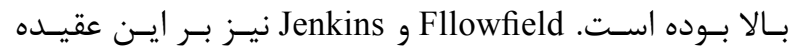

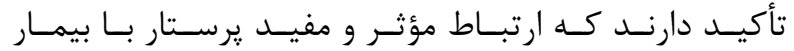

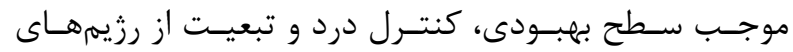

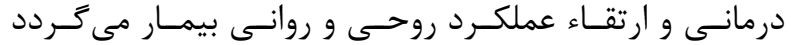

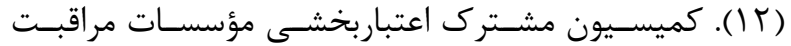

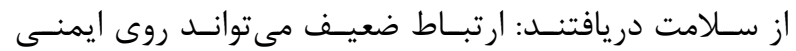

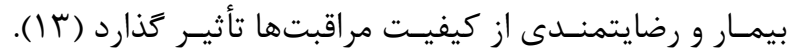

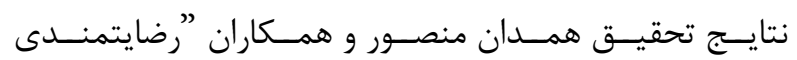

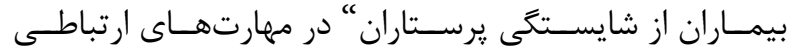

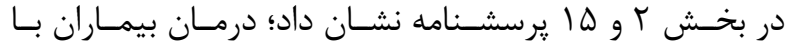

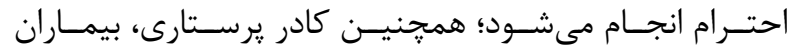

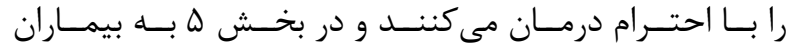

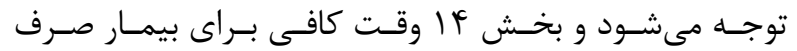

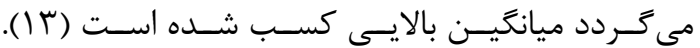

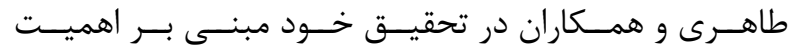

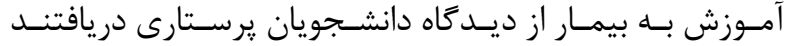

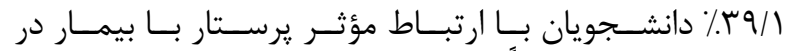

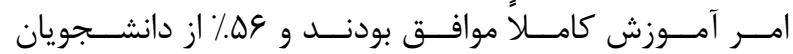

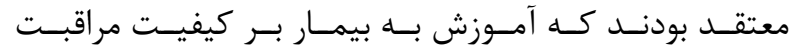

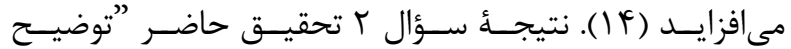

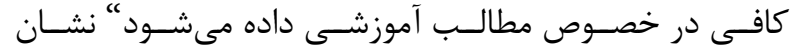

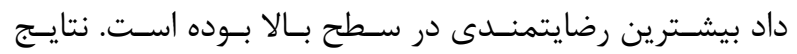

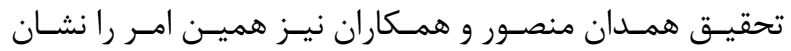

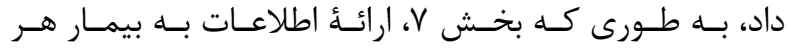

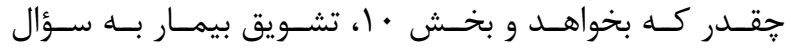

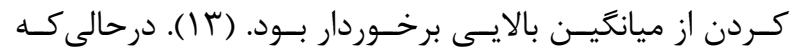

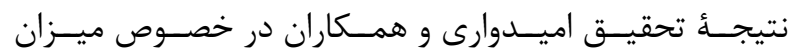

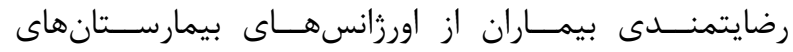

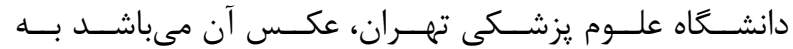

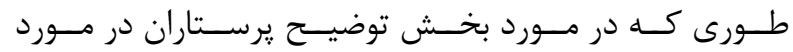

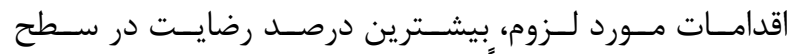

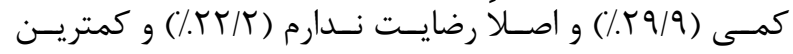

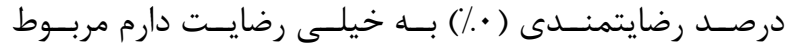
مىشــود (1) (1).

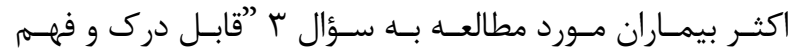

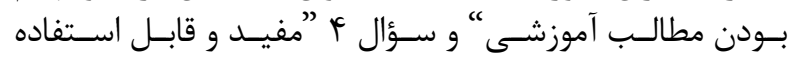

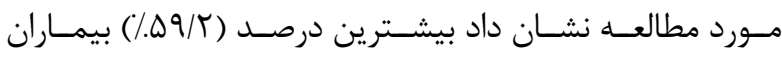

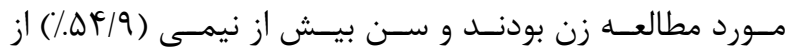

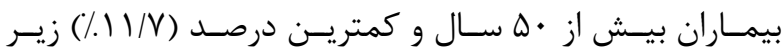

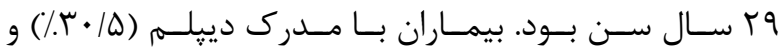

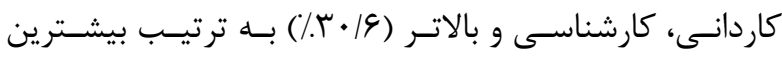

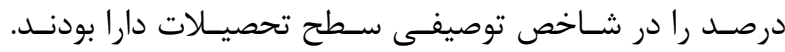

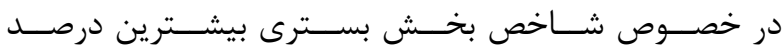

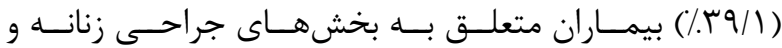

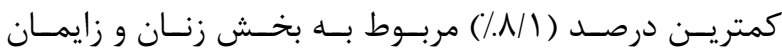

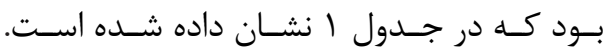

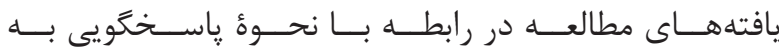

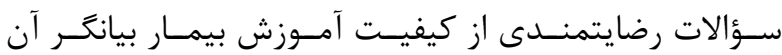

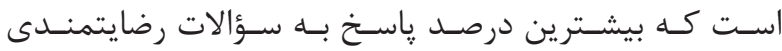

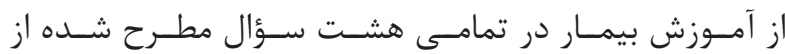

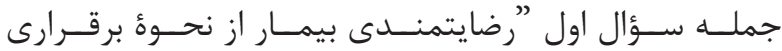

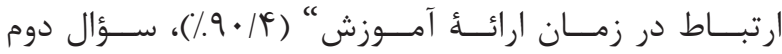

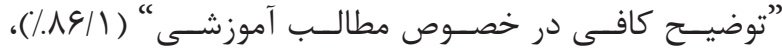

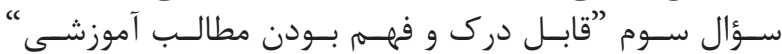

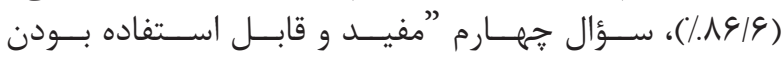

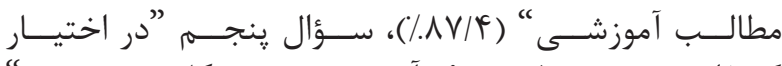

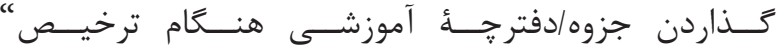

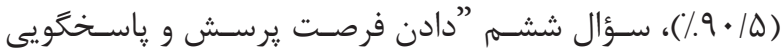

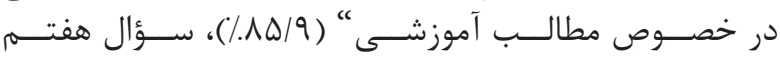

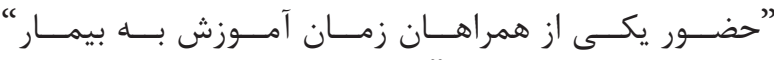

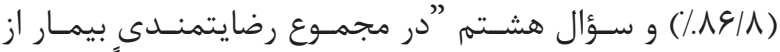

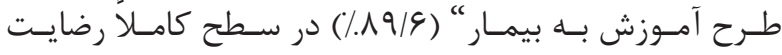

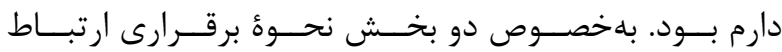

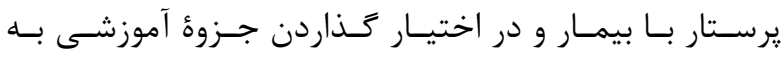

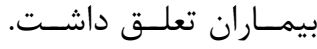

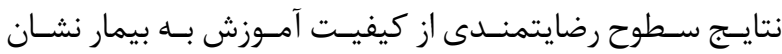

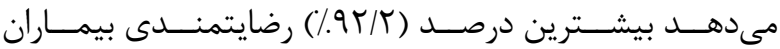

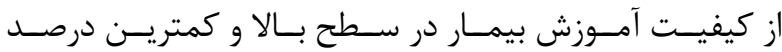

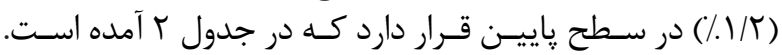

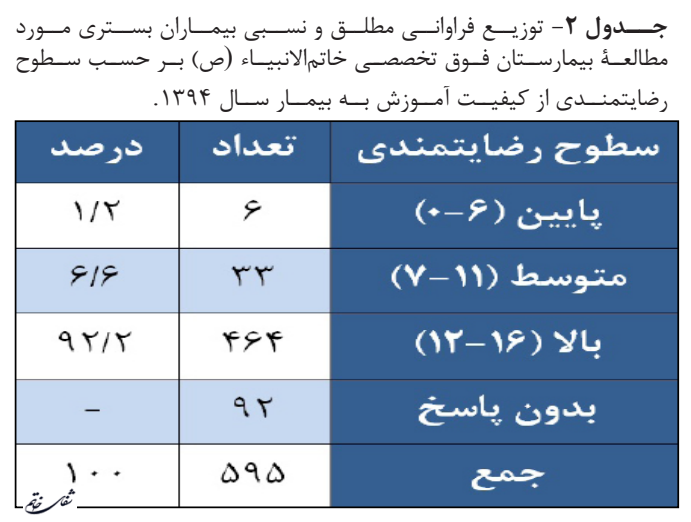




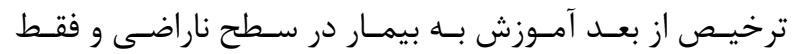

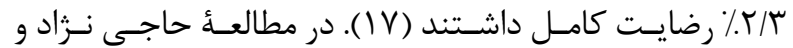

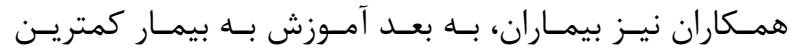

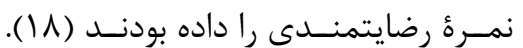

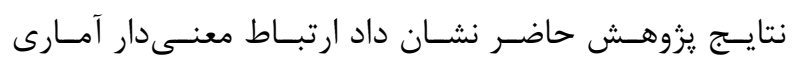

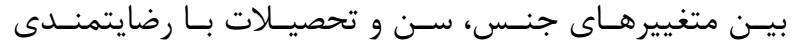

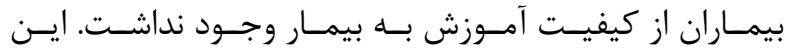

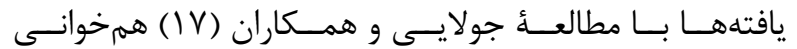

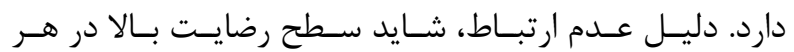

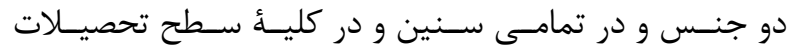

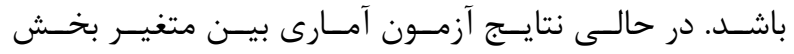

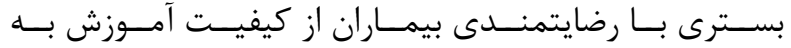

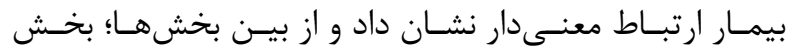

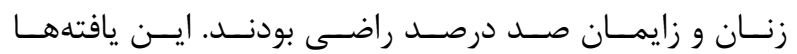

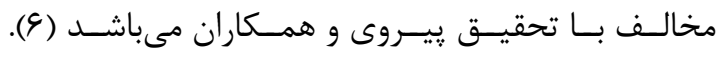

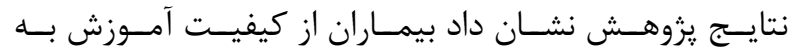

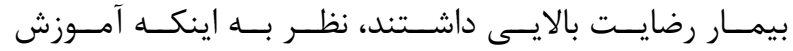

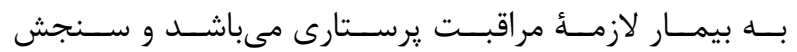

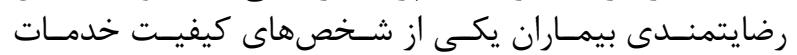

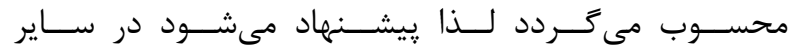

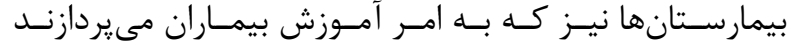

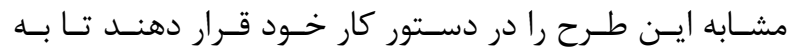

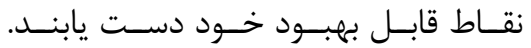

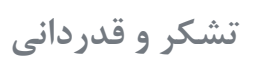

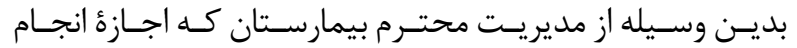

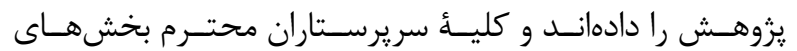

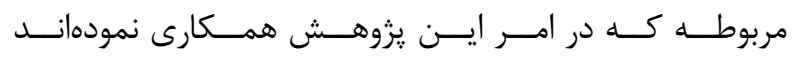

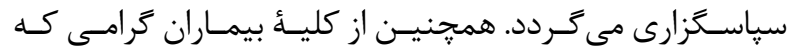

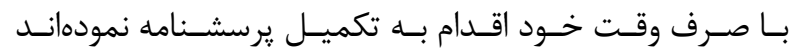

$$
\text { قدردانسى مى تَــردد. }
$$

1. Emami Razavi SH, Mohaghegh MR, Razavi SS. hospital accreditation standards in Iran. $1^{\text {st }}$ ed. Tehran: Seda Publication Center. 2000; p. 8-9, 32.

2. Farzianpour F, kalantari E, Rahimi Foroushani A, Alirezaei S. Guideline of assessment of Iran hospital accreditation standards. 2014; 76(5): 119-24.

3. Aghel-Kolsaraki N, Tol A, Heshmat R, Larijani B, Ghorbani M, Mohajeri-Tehrani M. Investigation of patient and employee satisfaction in endocrine and metabolism ward of center of education, research and treatment of Dr Shariati. Journal of Iran Diabetes \& Lipid.. 2011; 11(2): 154-61.

4. Jafar Jalal E, Joolaee S, Hajibabaee F, Bahrani ${ }^{2}$ Procedure

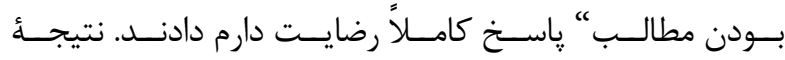

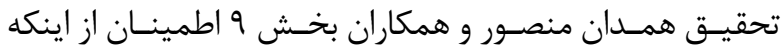

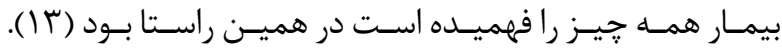

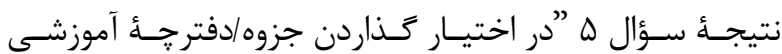

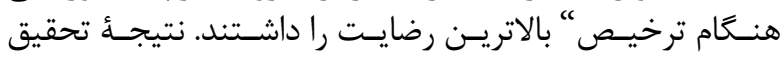

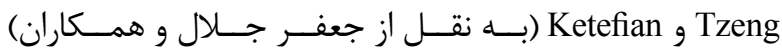

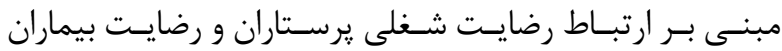

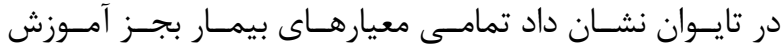

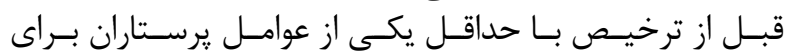

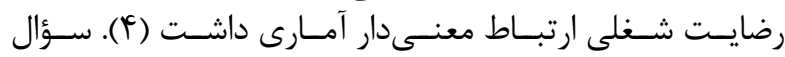

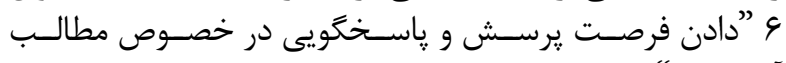

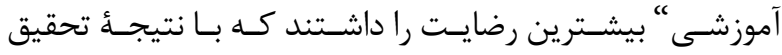

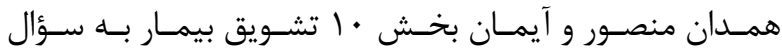

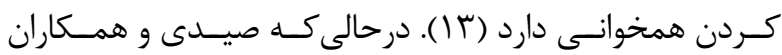

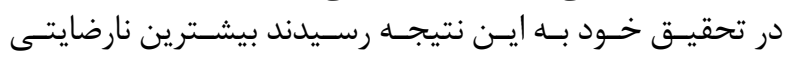

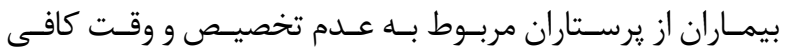

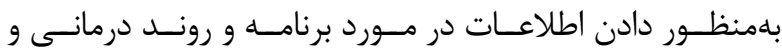

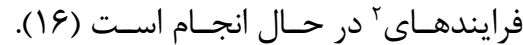

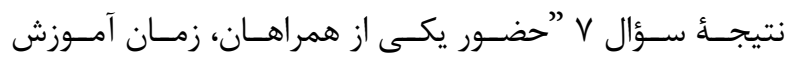

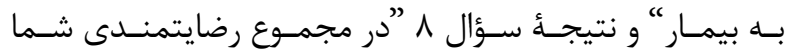

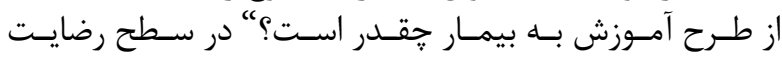

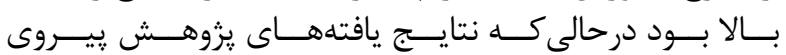

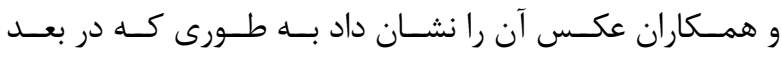

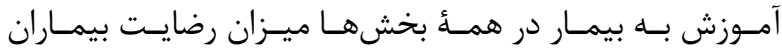

در حسد متوسـط بـود (9).

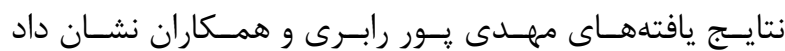

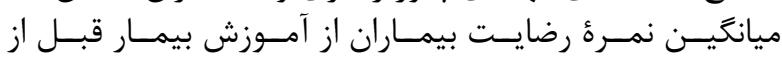

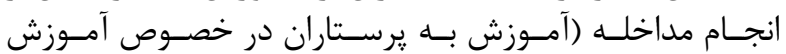

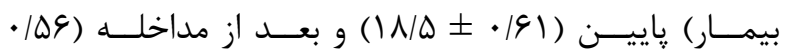

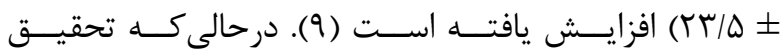

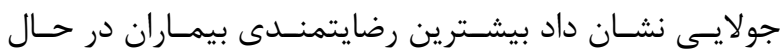

\section{منابع}

N. Evaluating the relationship between nurses' occupational satisfaction and patients' satisfaction with nursing service. IJNR. 2015; 10(1): 25-34.

5. Ahmad I, Sirajud Din. Patients' satisfaction from the health care services. GJMS. 2010; 8(1): 95-7.

6. Peyrovi H, Bahadori A, Ashghali FM, Haghani H. Comparison of in-patients, satisfaction with different domains of nursing care. Quarterly Journal of Nursing Management. 2013; 2(1): 59-66.

7. Prakash Bhanu. Patient satisfaction. Journal of Cutaneous and Aesthetic Surgery. 2010; 3(3): 151-5.

8. HSHS St. Joseph Hospital Highland. Hospital Sisters 
Health System. http://www.stjosephshighland.org/ourhospital/hospital-sisters-health-system/

9. Mehdi-pur Raberi R, Jamshidi N, Soltani Nejad A, Sabzevari S. Effects of nurse education on both patients' satisfaction of teaching patients, and nurses' knowledge, attitude and performance in intensive care units of teaching hospitals. Journal of Health and Care. 2011; 13(1): 0-0.

10. Toloei M, Dehghan nayeri N, Faghihzadeh S, Sadooghi-Asl A. The nurses' motivating factors in relation to patient training. Hayat. 2006; 12(2): 43-51.

11. Liu Y, Wang G. Inpatient satisfaction with nursing care and factors influencing satisfaction in a teaching hospital in China. J Nurs Care Qual. 2007; 22(3): 266-71.

12. Fllowfield L, Jenkins V. Effective communication skills are the key to good cancer care. Eur J Cancer. 1999; 35(11): 1592-7.

13. Hamdan-Mansour AM, Aboshaiqah AE, Thultheen IN, Salim WM, Azzeghaiby SN, Anani MA. Patients' satisfaction about nurses' competency in practicing communication skills. Life Science Journal. 2014; 11(3): 339-45.

14. Tahery N, Razavi Zadegan M, Kaiali M, Rashidi M,
Yaghoobi M, Shirzaei K, et al. Importance of patient education according abadan nursing student, opinions. Scientific Journal of Nursing and Midwifery School of Birjand University of Medical Sciences (Quarterly). 2011; 8(2): 100-6.

15. Omidvary S, Shahidzadeh-Mahani A, Mpntazery A, Azin SA, Harirchi AM, Soori H, et al. Patient satisfaction of emergency of hospitals of Tehran university of medical sciences. Payesh, Journal of the Iranian Institute for Health Sciences Research (Quarterly). 2008; 7(2): 141-52.

16. Seydi M, Heydari A, Khorami Rad A, Abbasi M. Investigation of patient satisfaction of intervention of islamic guideline in intervention (for the same sex) in Qom Kamkar and Nekoei hospitals of Qom university of medical science. 2nd international of medical congress of medical ethics in Iran- Tehran. 2008; p. 190-2.

17. Joolaee S, Hajibabaee F, Jafar Jalal E, Bahrani N. Assessment of patient satisfaction from nursing care in hospitals of Iran university of medical sciences. Hayat. 2011; 17(1): 35-44.

18. Hajinezhad MS, Rafii F, Jafarjalal E, Haghani H. Relationship between nurse caring behaviors from patients' perspectives and their satisfaction. IJN. 2007; 20(49): 73-83. 